\title{
Implementation of a Symptom-Triggered Protocol for Severe Alcohol Withdrawal Treatment in a Medical Step-down Unit
}

\author{
Paul W. Huang, MD, and Rohit Bhalla, MD, MPH
}

Objective: This single-center, quasi-experimental study of adult patients admitted or transferred to a medical stepdown unit with alcohol withdrawal diagnoses sought to determine if symptom-triggered therapy (STT) is more effective than combined fixed-scheduled (FS) and STT in severe alcohol withdrawal.

Methods: In the preintervention group (72 episodes), patients were treated with FS and STT based on physician preference. In the postintervention group (69 episodes), providers were required to utilize only the STT protocol.

Results: Implementation of the intervention was associated with a significant reduction in average (per patient) cumulative benzodiazepine dose, from $250 \mathrm{mg}$ to 96 $\mathrm{mg}(P<.001)$ and a decrease in average length of stay from 8.0 days to 5.1 days $(P<.001)$. Secondary safety measures included a reduction in the proportion of patients who experienced delirium tremens from $47.5 \%$ to $22.5 \%(P<.001)$, and a reduction in intubation rates from $13.8 \%$ to $1.3 \%(P=.003)$.

Conclusion: The STT protocol proved to be more effective and safer in treating severe alcohol withdrawal patients than usual care employing STT with FS. We believe the successful implementation of a STT protocol in high-acuity patients requires frequent monitoring to assess withdrawal severity combined with appropriate and timely dosing of benzodiazepines.

Keywords: alcohol withdrawal delirium; alcohol withdrawal syndrome; treatment protocol; benzodiazepine; lorazepam.
M anagement of severe alcohol withdrawal and delirium tremens (DT) is challenging and requires significant resources, including close monitoring and intensive treatment, frequently in an intensive care unit (ICU). ${ }^{1}$ Early diagnosis and therapeutic intervention are important to limit potential complications associated with DT. ${ }^{2}$ Benzodiazepines are first-line therapeutic agents, but the definition of optimal use and dosing regimens has been limited, due to a lack of randomized controlled trials. In lower acuity patients admitted to a detoxification unit, systematic symptom-triggered benzodiazepine therapy (STT) has been established to be more effective than fixed-schedule (FS) dosing. ${ }^{3-5}$ Patients treated using STT require lower total benzodiazepine dosing and achieve shorter treatment durations. However, in higheracuity patients admitted to general medical services, analyses have not shown an advantage of STT over combined FS and STT. ${ }^{6}$

\section{Methods}

The purpose of this study was to determine whether implementation of STT is more effective than FS dosing combined with episodic STT in the management of hospitalized high-acuity alcohol withdrawal patients. We conducted a preintervention and postintervention quasi-experimental study in the step-down unit (SDU) of a 305-bed community teaching hospital. The study population consisted of adult inpatients 18 years or older admitted or transferred to the 12-bed SDU with alcohol withdrawal, as defined by primary or secondary International Classification of Diseases, Tenth Revision diagnoses. SDU admission criteria included patients with prior DT or those who had received multiple doses of benzodiazepines in the emergency department. In-hospital transfer to the SDU was at the physician's discretion, if the patient required esca-

From Stamford Hospital, Stamford, CT. 
lating doses of benzodiazepines or the use of increasing resources, such as those for behavioral emergencies. The majority of patients admitted or transferred to the SDU were assigned to medical house staff teams under hospitalist supervision, and, on occasion, under community physicians. The nurse-to-patient ratio in the SDU was 1:3.

\section{Study groups}

The preintervention group consisted of 80 successive treatment episodes involving patients admitted or transferred to the SDU from December 2, 2015, to July 1, 2017. Patients were treated based upon physician preference, consisting of a scheduled dosing regimen with additional doses as needed. The postintervention group included 80 successive treatment episodes involving patients admitted or transferred to the SDU from October 1, 2017, to March 23,2019 . The STT protocol was used in all patients in the postintervention group.

In the preintervention group, fixed, scheduled doses of lorazepam or chlordiazepoxide and as-needed lorazepam were prescribed and adjusted based upon physician judgment. Monitoring of symptom severity was scored using the revised Clinical Institute Withdrawal Assessment for Alcohol scale (CIWA-Ar). Benzodiazepine dosing occurred if the CIWA-Ar score had increased 2 or more points from the last score.

In the postintervention group, the STT protocol included the creation of a standardized physician order set for benzodiazepine "sliding scale" administration. The STT protocol allowed for escalating doses for higher withdrawal scores. Symptom severity was scored using MINDS (Minnesota Detoxification Scale) criteria. ${ }^{1}$ Lorazepam as-needed dosing was based upon MINDS scores. A MINDS score less than 10 resulted in no medication, MINDS 10-12 required $2 \mathrm{mg}$, MINDS 13-16 required $4 \mathrm{mg}$, MINDS 17-19 required $6 \mathrm{mg}$, and MINDS 20 required $8 \mathrm{mg}$ and a call to the physician. Transfer to the ICU was recommended if the MINDS score was $\geq 20$ for 3 consecutive hours. Monitoring intervals occurred more frequently at 30 minutes unless the MINDS score was less than 10. After 7 days, the MINDS protocol was recommended to be discontinued, as the patient might have had iatrogenic delirium.

The STT protocol was introduced during a didactic session for the hospitalists and a separate session for internal medicine and family residents. Each registered nurse working in the SDU was trained in the use of the STT protocol and MINDS during nursing huddles.

Patients were excluded from evaluation if they were transferred to the SDU after 7 or more days in the hospital, if they had stayed in the hospital more than 30 days, were chronically on benzodiazepine therapy (to avoid confounding withdrawal symptoms), or if they left the hospital against medical advice (AMA). To avoid bias in the results, the patients with early discontinuation of treatment were included in analyses of secondary outcomes, thus resulting in all 80 episodes analyzed.

\section{Measures and data}

The primary outcome measure was benzodiazepine dose intensity, expressed in total lorazepam-equivalents. Secondary measures included average length of stay (including general medical, surgical, and ICU days), seizure incidence, DT incidence, sitter use, behavioral emergency responses, rates of leaving AMA, intubation, transfer to the ICU, and death.

Benzodiazepine dosing and length of stay were obtained from the data warehouse of the hospital's electronic health record (EHR; Meditech). Benzodiazepine dosing was expressed in total lorazepam-equivalents, with conversion as follows: lorazepam orally and intravenously $1 \mathrm{mg}$ = chlordiazepoxide $25 \mathrm{mg}$ = diazepam $5 \mathrm{mg}$. All other measures were obtained from chart review of the patients' EMR entries. The Stamford Hospital Institutional Review Board approved this study.

\section{Analysis}

Data analyses for this study were performed using SPSS version 25.0 (IBM). Categorical data were reported as frequency (count) and percent within category. Continuous data were reported as mean (SD). Categorical data were analyzed using $\chi^{2}$ analysis; continuous data were analyzed using t-tests. A $P$ value of .05 was considered significant for each analysis.

\section{Results}

During the preintervention period, 72 episodes (58 patients) met inclusion criteria, and 69 episodes (55 patients) met inclusion criteria during the postintervention period. Ten 
Table 1. Comparison of Demographic Characteristics by Preintervention and Postintervention Group

\begin{tabular}{|c|c|c|c|}
\hline & Preintervention & Postintervention & \\
\hline Characteristic & $\begin{array}{l}\text { Episodes, No. (\%) } \\
\qquad N=72\end{array}$ & $\begin{array}{l}\text { Episodes, No. (\%) } \\
\qquad N=69\end{array}$ & $P$ value \\
\hline \multicolumn{4}{|l|}{ Sex } \\
\hline Male & $64(88.9)$ & $62(89.9)$ & .85 \\
\hline Female & $8(11.1)$ & $7(10.1)$ & .85 \\
\hline History of withdrawal seizure & $32(44.4)$ & $26(37.7)$ & .42 \\
\hline History of DT & $36(50.0)$ & $29(42.0)$ & .34 \\
\hline Recent opiate use & $0(0.0)$ & $0(0.0)$ & NA \\
\hline Recent cocaine use & $1(1.4)$ & $3(4.3)$ & .33 \\
\hline Recent barbiturate use & $1(1.4)$ & $1(1.4)$ & .98 \\
\hline Age, mean ( \pm SD), y & $45 \pm 12$ & $44 \pm 13$ & .57 \\
\hline
\end{tabular}

DT, delirium tremens; NA, not applicable.

patients were represented in both groups. Eight preintervention episodes were excluded from the primary analysis because the patient left AMA. Eleven postintervention episodes were excluded: 9 due to patients leaving AMA, 1 due to chronic benzodiazepine usage, and 1 due to transfer to the SDU unit after 7 days. Baseline characteristics and medication use profiles of the preintervention and postintervention groups are summarized in Table 1.

Implementation of the intervention was associated with a significant reduction in average (per patient) cumulative benzodiazepine dose, from $250 \mathrm{mg}$ to $96 \mathrm{mg}$ $(P<.001)$, as shown in Table 2. Average length of stay decreased from 8.0 days to 5.1 days $(P<.001)$. Secondary safety measures were notable for a reduction in DT incidence, from $47.5 \%$ to $22.5 \%(P<.001)$, and lower rates of intubation, from $13.8 \%$ to $1.3 \%$ $(P=.003)$. Seven-day readmission rates were $0 \%$ preintervention and $1.4 \%$ postintervention.

\section{Discussion}

We found that hospitalized patients with severe alcohol withdrawal treated with STT required fewer benzodiazepines and had a lower length of stay than patients treated with a conventional combined STT and FS regimen. Implementation of the change from the STT and FS approach to the STT approach in the SDU resulted in concerns that waiting for symptoms to appear could result in more severe withdrawal and prolonged treatment. ${ }^{3}$ To address this, the intervention included monitoring and dosing every 30 minutes, as compared to monitoring and dosing every 1 hour preintervention. In addition, a sliding-scale approach to match alcohol withdrawal score with dosage was employed in postintervention patients.

Employment of the STT protocol also resulted in decreased complications, including lower rates of DT and transfer to the ICU. This new intervention resulted in significantly decreased time required to control severe symptoms. In the preintervention phase, if a patient's symptoms escalated despite administration of the as-needed dose of benzodiazepine, there was often a delay in administration of additional doses due to the time needed for nurses to reach a physician and subsequent placement of a new order. In the postintervention phase, the STT protocol allowed nursing staff to give benzodiazepines without delay when needed. We believe this reduced the number of calls by nursing staff to physicians requesting additional medications, and that this improved teamwork when managing these patients.

As part of the intervention, a decision was made to use the MINDS scale rather than the CIWA-Ar scale to assess withdrawal severity. This was because the CIWA-Ar has only been validated in patients with uncomplicated alcohol withdrawal syndrome and has not been researched extensively in patients requiring ICU-level care. ${ }^{1}$ MINDS 
Table 2. Comparison of Treatment Outcomes by Treatment Group

\begin{tabular}{lccc}
\hline & Preintervention & Postintervention & P value \\
\hline Primary outcomes & Episodes, No. (\%) & Episodes, No. (\%) & $\mathbf{N}=\mathbf{6 9}$ \\
\hline $\begin{array}{l}\text { Mean benzodiazepines per patient, } \\
\text { lorazepam equivalents (mg) }\end{array}$ & $250 \pm 188$ & $96 \pm 133$ & $<.001$ \\
\hline Secondary outcomes & $\mathbf{N}=\mathbf{8 0}$ & $\mathbf{N}=\mathbf{8 0}$ & $<.001$ \\
\hline Length of stay (days \pm SD) & $8.0 \pm 4.5$ & $5.1 \pm 4.1$ & .16 \\
\hline Seizures & $2(2.5)$ & $0(0.0)$ & $<.001$ \\
\hline DT & $38(47.5)$ & $18(22.5)$ & .13 \\
\hline Sitter usage & $14(17.5)$ & $22(27.5)$ & $>.99$ \\
\hline Request for trained team response & $15(18.8)$ & $15(18.8)$ & .80 \\
\hline Leaving AMA & $8(10.0)$ & $9(11.3)$ & .003 \\
\hline Intubation & $11(13.8)$ & $1(1.3)$ & .15 \\
\hline Transfer to ICU & $25(31.3)$ & $17(21.3)$ & NA \\
\hline Death & $0(0.0)$ & $0(0.0)$ & \\
\hline
\end{tabular}

AMA, against medical device; DT, delirium tremens; ICU, intensive care unit; NA, not applicable.

assessment has proven to be reliable and reflects severity of withdrawal. Furthermore, MINDS requires less time to administer -3 to 5 minutes vs 5 to 15 minutes for the CIWA-Ar scale. CIWA-Ar, unlike MINDS, requires subjective input from the patient, which is less reliable for higher acuity patients.

Our study is unique in that it focused on high-acuity patients and it showed both a significant reduction in quantity of benzodiazepines prescribed and length of stay. Previous studies on lower acuity patients in detoxification units have confirmed that STT is more effective than a FS approach. ${ }^{3-5}$ In patients of higher acuity, STT has not proven to be superior.

A key lesson learned was the need for proper education of nursing staff. Concurrent nursing audits were necessary to ensure that scoring was performed in an accurate and timely manner. In addition, it was challenging to predict which patients might develop DTs versus those requiring a brief inpatient stay. While there was initial concern that an STT protocol could result in underdosing, we found that patients had fewer DT episodes and fewer ICU transfers.

This study had several limitations. These include a relatively small sample size and the data being less recent. As there has been no intervening change to the therapeutic paradigm of DT treatment, the findings remain pertinent to the present time. The study employed a simple pre/post design and was conducted in a single setting. We are not aware of any temporal or local trends likely to influence these results. Admissions and transfers to the SDU for severe alcohol withdrawal were based on physician discretion. However, patient characteristics in both groups were similar (Table 1). We note that the postintervention STT protocol allowed for more frequent benzodiazepine dosing, though benzodiazepine use did decrease. Different alcohol withdrawal scores (MINDS vs. CIWA-Ar) were used for postintervention and preintervention, although previous research has shown that MINDS and CIWA-Ar scores correlate well. ${ }^{7}$ Finally, some patients of higher acuity and complexity were excluded, potentially limiting the generalizability of our results.

\section{Conclusion}

Our STT protocol proved to be more effective and safer in treating severe alcohol withdrawal patients than usual care employing STT with FS. We believe the successful 
implementation of a STT protocol in high-acuity patients also requires frequent monitoring using the MINDS scale, integrated with benzodiazepine sliding-scale dosing to match symptom severity. This bundled approach resulted in a significant reduction of benzodiazepine usage and reduced length of stay. Timely treatment of these patients also reduced the percent of patients developing DTs, and reduced intubation rates and transfers to the ICU. Further studies may be warranted at other sites to confirm the effectiveness of this STT protocol.

Corresponding author: Paul W. Huang, MD, Stamford Hospital, One Hospital Plaza, PO Box 9317, Stamford, CT 06904; phuang@ stamhealth.org.

Financial disclosures: None.

doi: 10.12788/jcom.0048

\section{References}

1. DeCarolis DD, Rice KL, Ho L, et al. Symptom-driven lorazepam protocol for treatment of severe alcohol withdrawal delirium in the intensive care unit. Pharmacotherapy. 2007;27(4):510-518.

2. DeBellis R, Smith BS, Choi S, Malloy M. Management of delirium tremens. J Intensive Care Med. 2005;20(3):164-173.

3. Saitz R, Mayo-Smith MF, Roberts MS, et al. Individualized treatment for alcohol withdrawal. A randomized double-blind controlled trial. JAMA. 1994;272(7):519-523.

4. Sachdeva A, Chandra M, Deshpande SN. A comparative study of fixed tapering dose regimen versus symptom-triggered regimen of lorazepam for alcohol detoxification. Alcohol Alcohol. 2014;49(3):287-291.

5. Daeppen JB, Gache P, Landry U, et al. Symptom-triggered vs fixed-schedule doses of benzodiazepine for alcohol withdrawal: a randomized treatment trial. Arch Intern Med. 2002;162(10):1117-1121.

6. Jaeger TM, Lohr RH, Pankratz VS. Symptom-triggered therapy for alcohol withdrawal syndrome in medical inpatients. Mayo Clin Proc. 2001;76(7):695-701.

7. Littlefield AJ, Heavner MS, Eng CC, et al. Correlation Between mMINDS and CIWA-Ar Scoring Tools in Patients With Alcohol Withdrawal Syndrome. Am J Crit Care. 2018;27(4):280-286.

\section{CALL FOR MANUSCRIPTS}

- Allergy and

immunology

- Cardiovascular disease

- Diabetes mellitus

- Geriatrics and gerontology

- Gl disorders

- Hematology/ oncology

- HIV/AIDS

- Kidney disease

- Mental health

- Pain management

- Population health

- PTSD

- Pulmonary disease

- Rheumatology

- Stroke

- Substance abuse www.mdedge.com/jcomjournal/page/information-authors.
Email your manuscript or queries to eseger@mdedge.com. 